\title{
Semiclassical Casimir Energies at Finite Temperature
}

\author{
Martin Schaden ${ }^{1,2}$ and Larry Spruch ${ }^{1}$ \\ ${ }^{1}$ New York University, Physics Department \\ 4 Washington Place, New York, New York 10003 \\ ${ }^{2}$ Union College*, Physics Department \\ Science and Engineering Bldg., Schenectady, New York, 12308
}

October 29, 2018

\begin{abstract}
We study the dependence on the temperature $T$ of Casimir effects for a range of systems, and, in particular, for a pair of ideal parallel conductors, $l_{1}$ by $l_{2}$, separated by a vacuum, a distance $l_{3}$ apart, with $l_{1} \gg l_{3}$ and $l_{2} \gg l_{3}$. We study the Helmholtz free energy $A^{T}$, combining Matsubara's formalism, in which the temperature $T$ appears as a periodic Euclidean fourth dimension of circumference $l_{T}=\hbar c / k_{B} T$ with the semiclassical periodic orbital approximation of Gutzwiller. The latter was shown to be exact for parallel plates at $T=0$. By inspecting the known results for the Casimir energy at $T=0$ in two cases of a rectangular parallelepiped, $\left(l_{1} \gg l_{3}\right.$ and $l_{2} \gg l_{3}$, and $l_{1} \gg l_{2}$ and $\left.l_{1} \gg l_{3}\right)$, one is led to guess at the expression for $A^{T}$ of two ideal parallel conductors, without performing any calculation. The result is a new form for $A^{T}$, namely, $A^{T}=-\left(2 \hbar c l_{1} l_{2} l_{3} / \pi^{2}\right) \sum_{n_{3}=1}^{\infty} \sum_{n_{T}=-\infty}^{\infty} L^{-4}\left(n_{3}, n_{T}\right)$, where $L\left(n_{3}, n_{T}\right)=\left[\left(2 n_{3} l_{3}\right)^{2}+\left(n_{T} l_{T}\right)^{2}\right]^{1 / 2}$ is the length of a classical periodic path on a two-dimensional cylinder section. This expression for the free energy is equivalent to others that have been obtained in the literature. At $T=0$ the semiclassical approach provides a finite and systematic approximation scheme in terms of classical paths that is useful when the normal modes of the cavity cannot be determined either explicitly or implicitly. Slightly extending the domain of applicability of Gutzwiller's semiclassical periodic orbit approach, we here evaluate the free energy at $T>0$ in terms of periodic classical paths in a four-dimensional cavity that is the tensor product of the original cavity and a circle. The validity of this approach is at present restricted to particular systems. We also discuss the origin of the classical ( $\hbar$-independent) form of $A^{T}$ for $T \sim \infty$.
\end{abstract}

Key Words: Casimir energies, semiclassical theory, periodic orbits, finite temperature

*Present address. Email address: schadenm@union.edu 


\section{INTRODUCTION}

The best known of the Casimir effects is the force per unit area between two parallel ideal plates, separated by vacuum, a distance $l_{3}$ apart, at a temperature $T=0$ [1. Some fifty years later, that and other Casimir effects continue to intrigue both theoreticians and experimentalists. Indeed, interest has been growing. We list a few general references 2, 3, 4, 5. Of particular interest to us here are recent experimental reports by Lamoreaux[6] and by Mohideen and colleagues/7. Very recently the possible role of this effect in microelectromechanical systems has been examined [8]. To avoid alignment problems one studies the force between a sphere of radius $R$ and a wall, where the point on the sphere closest to the wall is at a distance $l$ from the wall, with $l \ll R$. (The analysis of the force in this case is intimately connected to the force between walls [2, 3, 9] and the experiment is simpler to perform. An experiment which might be even simpler to perform, because the force would be greater, is the force between a sphere and a segment of a spherical shell. We are here dealing with the focusing of virtual photons [10]. Focusing of virtual photons by a parabolic mirror has also been considered[11].) For the values of $R$ and $l$ studied, the accuracy was such that a number of corrections to the case of ideal conductors must be considered. In particular, finite temperature effects must be accounted for.

The corrections to the Casimir force for $T>0$ were first studied in a seminal paper by Lifshitz 12 in 1956. The paper studied dielectric slabs, and the temperature effects were a matter of dispute for years. The subject was first clarified in 1978 13. It was shown that Lifshitz's basic results for the temperature corrections were correct, but that Lifshitz had erred in taking the limit as the dielectric constant goes to infinity when going from a dielectric to an (ideal) conductor.

Our primary interest will be in rederiving the temperature effect for ideal walls but in a new form. We will utilize two sets of results, one by Matsubara 14 for accounting for temperature effects in a general setting, and the second a formulation of Casimir effects at $T=0$ in terms of semiclassical extremal paths by the present authors 15 . The reformulation has the merit that the dominant paths are often rather obvious, with contributions that can be evaluated even when the proper frequency modes cannot be determined.

We take this opportunity to mention two significant papers 16 by Balian and Duplantier not referred to in 15]. The methods used in 16] were quite different

from those used in our paper. We had not fully appreciated the two papers at the time 15 was published.

\section{IDEAL PARALLEL PLATES: A HEURISTIC APPROACH}

Matsubara showed 14] that in studying a system in three-dimensional space in equilibrium at temperature $T$, it can be convenient to consider the system in a four-dimensional Euclidean space whose fourth dimension at finite temperature $T>0$ is compactified to a circle of circumference

$$
l_{T}=\hbar c /\left(k_{B} T\right) .
$$


Thus, consider two ideal parallel conductors at temperature $T$, with an area $\mathcal{A}=l_{1} l_{2}$ separated a distance $l_{3}$ in vacuum with $l_{3} \ll l_{1}$ and $l_{3} \ll l_{2}$. The force between the plates in this case is proportional to $l_{1}$ and $l_{2}$ and the force per unit area does not depend on either dimension. At finite temperature, the essential geometry in this case is that of a two-dimensional cylindrical surface of length $l_{3}$ and circumference $l_{T}$. (See App. A)

In our study 15. of the plates at $T=0$ the analysis involved the determination of the difference, $\Delta \rho(E)$, of the spectral densities in the presence and absence of the conductors. Gutzwiller [17] showed that this quantity is given in semi-classical approximation by the periodic paths of extremal classical action, in our case the periodic paths of extremal length. (Note that none of these paths has zero length and that a stationary periodic path must involve a minimum of two reflections.) In the case of parallel plates these are classical paths going back and forth between, and perpendicular to, the walls.

The analogous analysis at finite temperature would involve studying the semiclassical Green function with a periodic dependence on the Euclidean time. The initial and final space-time points are still the same, but the Euclidean geometry is that of a cylinder. We prefer to proceed differently.

For $T>0$ (and fixed volume), it is natural to work with the Helmholtz free energy $A^{T}$ rather than with $\mathcal{E}^{T}$, the (Casimir) energy itself, and there is a natural choice of the form of $A^{T}$. Thus, abandoning any pretense of rigor, we guess at the form of $A^{T}$ - there remains the determination of an over-all numerical factor - and justify our choice of $A^{T}$ afterwards.

The semi-classical path approach has advantages that go well beyond providing a simple physical picture of the origin of the Casimir effect at $T>0$; as noted earlier, one can often determine the significant paths even when the natural frequency modes cannot be determined (neither explicitly nor, as in the case of the generalized argument theorem[18, 19], implicitly).

We have

$$
A^{T}=A^{T}\left(\hbar, c, l_{1}, l_{2}, l_{3}, l_{T}\right) .
$$

At $T=0$, we were concerned with the energy density. At $T>0$, we will be concerned with the free energy density. In both cases we explicitly extract the volume $l_{3} \mathcal{A}$ of the cavity. Further, on dimensional grounds, $A^{T}$ is proportional to $\hbar c$. We can therefore write

$$
A^{T}=\hbar c l_{3} \mathcal{A} g\left(l_{3}, l_{T}\right),
$$

where $g$ is proportional to the inverse fourth power of a length. Now, in 15$]$ it was shown that for a cavity with $l_{3} \ll l_{1}, l_{2}, \mathcal{E}_{\text {Cas }}$ is proportional to $\sum_{n_{3}} L^{-4}\left(n_{3}\right)$, where the length $L\left(n_{3}\right)=2 n_{3} l_{3}$ and $n_{3}= \pm 1, \pm 2, \ldots$. Further, for a parallelepiped with $l_{3} \ll l_{1}$ and $l_{2} \ll l_{1}, \mathcal{E}_{\text {Cas }}$ was proportional to $\sum_{n_{2}} \sum_{n_{3}} L^{-4}\left(n_{2}, n_{3}\right)$, where $L\left(n_{2}, n_{3}\right)=\left[\left(2 n_{2} l_{2}\right)^{2}+\left(2 n_{3} l_{3}\right)^{2}\right]^{1 / 2}$ and the sum is over all pairs of integers $\left(n_{2}, n_{3}\right)$, positive, negative, and zero, other than $(0,0) . L\left(n_{3}\right)$ is the length of a classical periodic path perpendicular to the walls and reflected $\left|n_{3}\right|$ times from each of them. Positive values of $n_{3}$ refer to paths which start to the right, while negative values of $n_{3}$ refer to paths which start to the left. $L\left(n_{2}, n_{3}\right)$ is the length of a classical periodic path with $\left|n_{2}\right|$ reflections off each of the walls with dimensions $l_{1}$ by $l_{3}$ and $\left|n_{3}\right|$ reflections off each of the walls with dimensions $l_{1}$ by $l_{2}$. For the case with $l_{3} \ll l_{1}$ and $l_{3} \ll l_{2}, T>0$, to which we henceforth largely restrict our attention, the knowledge that the space is enlarged by one 
extra Euclidean dimension strongly suggests that $A^{T}$ can be obtained by simply replacing $L\left(n_{2}, n_{3}\right)$ by

$$
L\left(n_{3}, n_{T}\right)=\left[\left(2 n_{3} l_{3}\right)^{2}+\left(n_{T} l_{T}\right)^{2}\right]^{1 / 2}
$$

This length is that of a classical periodic extremal path on the surface of a cylinder of height $l_{3}$ and circumference $l_{T}$. The path starts in a given direction and reflects $\left|n_{3}\right|$ times from each of the ends of the cylinder section (the ideal walls) and circles the cylinder in a particular direction $\left|n_{T}\right|$ times. (The absence of a factor of 2 in the $n_{T} l_{T}$ term of Eq. (4) is due to the fact that we are considering extremal paths on a section of a cylindrical surface. See App. A) Negative values of $n_{3}$ refer to paths that start in the opposite direction, while negative values of $n_{T}$ refer to paths that circle the cylinder in the opposite direction. The contribution from the $n_{3}=0$ path can be ignored since it gives an (extensive) contribution to the free energy that is proportional to the volume; it does not contribute to the (net) force on a plate. [See paragraph in Sec. 4 above the paragraph which contains Eq. (21).]

We thus guess that

$$
A^{T}=K \hbar c \mathcal{A} l_{3} \sum_{\left(n_{3}, n_{T}\right)} L^{-4}\left(n_{3}, n_{T}\right)
$$

where the sum extends over all pairs of integers $\left(n_{3}, n_{T}\right)$ with $n_{3} \neq 0$, the length $L\left(n_{3}, n_{T}\right)$ is given by Eq. (4), and $K$ is a numerical coefficient. We determine the value of $K$ by demanding that $A^{T}$ reduce to

$$
\mathcal{E}_{\text {Cas }}=-\frac{\pi^{2} \hbar c \mathcal{A}}{720 l_{3}^{3}}
$$

for $T \sim 0$. But $T \sim 0$ implies that $l_{T} \sim \infty$ which in turn implies that only $n_{T}=0$ contributes to the sum in Eq. (5). For $T \sim 0$ we thus have

$$
A^{T \sim 0}=K \hbar c \mathcal{A} l_{3} 2 \sum_{n_{3}=1}^{\infty} \frac{1}{\left(2 n_{3} l_{3}\right)^{4}}=\frac{\pi^{4} K \hbar c \mathcal{A}}{720 l_{3}^{3}} .
$$

(The factor of 2 reflects the fact that we are now summing over positive values only of $n_{3}$.) Comparison with Eq. (6) gives $K=-1 / \pi^{2}$ and we obtain

$$
A^{T}=-\frac{2 \hbar c \mathcal{A} l_{3}}{\pi^{2}} \sum_{n_{3}=1}^{\infty} \sum_{n_{T}=-\infty}^{\infty} L^{-4}\left(n_{3}, n_{T}\right) .
$$

In App. B we show that Eq. (8) is indeed correct by comparing to a previous result. A proof from first principles, for a scalar field, is given in Sec. 5. The force per unit area at a temperature $T$ is then given by

$$
\frac{F^{T}}{\mathcal{A}}=\frac{1}{\mathcal{A}} \frac{\partial}{\partial l_{3}} A^{T}=-\frac{2 \hbar c}{\pi^{2}} \sum_{n_{3}=1}^{\infty} \sum_{n_{T}=-\infty}^{\infty} \frac{3\left(2 n_{3} l_{3}\right)^{2}-\left(n_{T} l_{T}\right)^{2}}{\left[\left(n_{T} l_{T}\right)^{2}+\left(2 n_{3} l_{3}\right)^{2}\right]^{3}}
$$

The somewhat complicated form of $F^{T}$ as opposed to the relatively simple form of $A^{T}$ shows that the free energy is not just a more fundamental object of study but also a more transparent one. Given $A^{T}, F^{T}$ follows as in Eq. (9). 


\section{THE HIGH-T AND LOW-T EXPANSIONS}

The free energy $A^{T}$ is readily obtained from Eq. (8) for $z$ large or small, where

$$
z=2 l_{3} / l_{T}=2 l_{3} k_{B} T /(\hbar c) .
$$

Isolating the $n_{T}=0$ contribution, the free energy of Eq. (8) may be rewritten in the form

$$
\frac{A^{T}\left(l_{3}\right)}{\mathcal{A}}=-\frac{\pi^{2} \hbar c}{720 l_{3}^{3}}-\frac{\left(k_{B} T\right)^{2}}{\pi^{2} \hbar c l_{3}} \Delta(z) .
$$

From Eq. (8) and the definition Eq. (ब) we have that the dimensionless function $\Delta(z)$ is

$$
\Delta(z)=\sum_{n=1}^{\infty} \sum_{m=1}^{\infty}\left[m^{2} z+n^{2} / z\right]^{-2}=\Delta(1 / z) .
$$

[To check Eq. (12), let $z \rightarrow 1 / z$, and interchange $m$ and $n$.] The reflection property [20], $\Delta(z)=\Delta(1 / z)$, is quite powerful. It relates the high- and lowtemperature regimes, or, equivalently, the large and small $l_{3}$ regimes. The expansion of $\Delta(z)$ for $z \gg 1$ is found by using the identity 21]

$$
\sum_{n=1}^{\infty} \frac{1}{n^{2}+a^{2}} \equiv J(a) / a=\frac{1}{2 a^{2}}(a \pi \operatorname{coth}(a \pi)-1)=-\frac{1}{2 a^{2}}+\frac{\pi}{2 a}+\frac{\pi}{a} \sum_{n=1}^{\infty} e^{-2 \pi n a} .
$$

Differentiating Eq. (13) with respect to $a^{2}$ and then replacing $a$ by $m z$ one obtains, after multiplication by $z^{2}$ and summation over $m$,

$$
\begin{aligned}
\Delta(z) & =\sum_{m=1}^{\infty}\left[-\frac{1}{2 m^{4} z^{2}}+\frac{\pi}{4 m^{3} z}+\frac{\pi}{2 m^{3} z} \sum_{n=1}^{\infty}(1+2 \pi m n z) e^{-2 \pi m n z}\right] \\
& =-\frac{\pi^{4}}{180 z^{2}}+\frac{\pi}{4 z} \zeta(3)+\frac{\pi}{2 z} \sum_{n=1}^{\infty} e^{-2 \pi n z}(1+2 \pi n z) \sum_{n / m} \sum_{\text {integer }>0} m^{-3},
\end{aligned}
$$

where $\zeta(3)=\sum_{m=1}^{\infty} m^{-3}=1.202 \ldots$.. Using the expansion of Eq. (14) in Eq. (11) gives for the free energy per unit area, for $2 l_{3} k_{B} T>\hbar c$, or $2 l_{3}>l_{T}$,

$$
\frac{A^{T}}{\mathcal{A}}=-\frac{\zeta(3) k_{B} T}{8 \pi l_{3}^{2}}-\frac{\left(k_{B} T\right)}{l_{T} l_{3}}\left(1+\frac{l_{T}}{4 \pi l_{3}}\right) e^{-4 \pi l_{3} / l_{T}}+O\left(\frac{k_{B} T}{l_{3}^{2}} e^{-8 \pi l_{3} / l_{T}}\right) .
$$

Using the expansion of Eq. (14) with $z$ replaced by $1 / z$ in Eq. (11) gives, for

$2 l_{3} k_{B} T<\hbar c$, or $2 l_{3}<l_{T}$,

$\frac{A^{T}}{\mathcal{A}}=-\frac{\pi^{2} \hbar c}{720 l_{3}^{3}}-\frac{k_{B} T}{2 \pi l_{T}^{2}} \zeta(3)+\frac{\pi^{2} k_{B} T l_{3}}{45 l_{T}^{3}}-\frac{k_{B} T}{l_{T} l_{3}}\left(1+\frac{l_{3}}{\pi l_{T}}\right) e^{-\pi l_{T} / l_{3}}+O\left(\frac{k_{B} T}{l_{T} l_{3}} e^{-2 \pi l_{T} / l_{3}}\right)$.

These low-T ( Eq. (16)) and high-T ( Eq. (15)) expansions of $A^{T} / \mathcal{A}$, first obtained by Sauer, by Mehra, and by Levin and Rytov[22, have also been reproduced in 13. (The present derivation is somewhat simpler, to some extent because some of the earlier derivations were in a broader context, with dielectric walls or rather arbitrary surfaces. Note that Mehra 22 uses a convention for which an attractive force between the plates is taken to be positive.) See[3] 
for a physical explanation of the contribution proportional to $T^{4}$ in the low- $\mathrm{T}$ expansion Eq. (16) of the free energy.

The expansions Eq. (16) and Eq. (15) are both exponentially good and the two limits are "dual" in the sense that since $\Delta(z)=\Delta(1 / z)$, they both follow from Eq. (14). The relative error of these approximations is largest at the transition point, $2 l_{3} k_{B} T=\hbar c$, or $z=2 l_{3} / l_{T}=1$. (The $l_{3}$ dependence of $A^{T} / \mathcal{A}$ is not well defined at the transition point, since, with $n$ arbitrary, a factor of $\left(2 l_{3} / l_{T}\right)^{n} \sim 1$ does not change the function appreciably near this point.) The exponential corrections all have the same sign and are no greater than $\sim 2.3 \%$ at any value of $l_{3} k_{B} T$. Perhaps most significant experimentally is that the high-temperature limit is valid for $l_{3}$ rather large compared to $\hbar c /\left(2 k_{B} T\right)$. This implies that the fall-off of the Casimir force at room temperature is proportional to $l_{3}^{-3}$ rather than $l_{3}^{-4}$ for separations $l_{3}$ rather large compared to 4 microns. Since the largest separation between the plates of early experiments was a few microns, it appears unlikely that a Casimir force proportional to $1 / l^{4}$ could have been observed over the entire range. It should, however, be recalled that we have here considered an ideal metal rather than a real one. On the other hand, corrections due to finite conductivity diminish with increasing separation and are expected to be negligible compared to the temperature correction for separations exceeding a few microns 3,23 .

\section{THE CLASSICAL FORM OF $A^{T}$ FOR $T \rightarrow \infty$}

The fact that for parallel plates $A^{T}$ at sufficiently large $T$ assumes a classical ( $\hbar$-independent) form, as seen in Eq. (15), warrants some comment. We begin with the remark that $A^{T}$ in the high-T limit is not generally $\hbar$-independent. Thus, for a thin smooth closed surface, one finds 16

$$
A^{T}=-\sigma k_{B} T \ln \left(k_{B} T l / \hbar c\right),
$$

where the dimensionless quantity $\sigma$ is a function of ratios of the radii of curvature and $l$ is an appropriately defined overall length scale. The logarithmic dependence of Eq. (17) on the overall length scale and $k_{B} T / \hbar c$ implies that the change of the free-energy does not depend on $\hbar c$ when all lengths are rescaled by the same amount, i.e., the "breathing mode" is always classical at high temperatures.

The fact that the free energy is a pure power law for parallel plates makes that case a bit special. If we assume that the high-T limit of the free energy of two parallel plates is classical, the power law dependence of $A^{T}$ follows on dimensional grounds. (It follows from $A^{T}=A^{T}\left(k_{B} T, l_{1}, l_{2}, l_{3}\right)$ that $A^{T}=k_{B} T l_{1} l_{2} g\left(l_{3}\right)$, where $g\left(l_{3}\right)$ must be proportional to $1 / l_{3}^{2}$.) One may argue for the existence of a classical high-T limit (and the absence of logarithmic corrections) from the contribution to the free energy of a single cavity mode of frequency $\omega_{k}$. Writing

$$
A^{T}=-\beta^{-1} \ln Z=\sum_{k} A_{k}^{T}=\sum_{k}\left[\frac{\hbar \omega_{k}}{2}+\beta^{-1} \ln \left(1-e^{-\beta \hbar \omega_{k}}\right)\right],
$$

$A_{k}^{T}$ for frequencies $\omega_{k} \ll k_{B} T / \hbar$ becomes

$$
A_{k}^{T \sim \infty}=\frac{\hbar \omega_{k}}{2}+\beta^{-1} \ln \left[\beta \hbar \omega_{k}\left(1-\frac{\beta \hbar \omega_{k}}{2}+O\left(\left(\beta \hbar \omega_{k}\right)^{2}\right)\right)\right] \sim k_{B} T \ln \left(\frac{\hbar \omega_{k}}{k_{B} T}\right),
$$


ignoring terms of order $\beta\left(\hbar \omega_{k}\right)^{2}$. Inserting the high-T expression Eq. (19) in Eq. (18) formally gives an asymptotic expansion of $A^{T}$. But since the frequencies of a cavity generally can be arbitrarily high and the neglected terms are not small for all frequencies at a fixed value of $T$, this expansion is valid only in the presence of a cutoff. However, for the change of the free energies of the cavity in two different configurations the mode sum is finite if the two configurations can be connected adiabatically, as, for instance, by moving an internal boundary arbitrarily slowly. An asymptotic expansion for large $T$ of this difference does make sense. Under an adiabatic change of boundary conditions, the eigenmodes of the cavity change from $\omega_{k}$ to $\omega_{k}^{\prime}$, but the quantum numbers of a mode are conserved. Using Eq. (19) one obtains for the asymptotic difference of the free energies when $T \sim \infty$

$$
\Delta A^{T}=A^{\prime T}-A^{T} \sim k_{B} T \sum_{k}\left[\ln \left(\frac{\omega_{k}^{\prime}}{\omega_{k}}\right)\right] .
$$

The sum in Eq. (20) converges if the difference of the free energies of the two configurations has a finite limit at $T=0$, since a finite temperature does not induce new ultraviolet divergences. The expression Eq. (20) for the difference of the free energies at sufficiently high temperature (or better, for $k_{B} T l \gg \hbar c$, where $l$ is a relevant length scale of the problem) is classical and notably does not depend on $\hbar$. In this limit the entropy $S$ of the cavity is $S=-A^{T} / T$ and Eq. (20) states that the difference in the entropies of a cavity at high temperatures approaches a constant that does not depend on the quantum scale $\hbar$. (That the entropy of a bosonic gas in a cavity has a finite limit as $T \rightarrow \infty$ is compatible with dimensional reduction, that is, the high temperature limit is described by Euclidean quantum field theory in 3 dimensions with $\hbar$ replaced by $i \beta[14$.)

Although plausible, it is not possible to prove the existence of a finite high temperature limit using only classical arguments, because one can only compare the energy $k_{B} T$ with the frequencies of the cavity by introducing a quantity with the dimension of $\hbar$. If the asymptotic limit exists, and we have just argued that it does, it is independent of the value of $\hbar$ one has chosen to make this comparison. On dimensional grounds, the classical contribution to the change in entropy from a single (independent) mode can then depend only on the frequency ratio. Further, since the contribution to the (change in) entropy of (independent) modes is additive, classically the (change) in entropy due to a single mode is proportional to $\ln \left(\omega^{\prime} / \omega\right)$. Eq. (20) then follows as the classical change in the free energy of a cavity from the definition of $k_{B}$.

In studying the force between $l_{1}$ by $l_{2}$ plates at a separation $l_{3}$ one can evaluate the energy in the volume between the plates and in the infinite volume outside the plates and then determine the force by differentiation with respect to $l_{3}$. It is a standard practice to avoid infinite volumes by considering a box of fixed volume $l_{1} \times l_{2} \times L$ with an additional (movable) plate parallel to the $l_{1}$ by $l_{2}$ walls at a distance $l_{3}$ from one of the walls [24. The total free energy of the box is the sum of the free energies of each of the two subvolumes: $A_{t o t}^{T}\left(l_{1}, l_{2}, L ; l_{3}\right)=A^{T}\left(l_{1}, l_{2}, l_{3}\right)+A^{T}\left(l_{1}, l_{2}, L-l_{3}\right)$. The force on the additional wall is $-\partial A_{t o t}^{T} / \partial l_{3}$. Extensive contributions to the free energy of the subsystems (proportional to their volume) do not contribute to the dependence of $A_{t o t}^{T}$ on

\footnotetext{
${ }^{1}$ There should be no confusion with our usage of $L$ to also denote path lengths.
} 
$l_{3}$ and therefore do not result in a (net) force on the middle wall. Note that generally only the difference of the free energy of the box compared to the free energy of the box in some "standard" configuration, (say with the additional wall at $\left.l_{3}=L / 2\right)$ i.e., $A_{d i f f}^{T}\left(l_{1}, l_{2}, L ; l_{3}\right)=A_{\text {tot }}^{T}\left(l_{1}, l_{2}, L ; l_{3}\right)-A_{\text {tot }}^{T}\left(l_{1}, l_{2}, L ; L / 2\right)$, is finite.

With these arguments, we can, purely classically, obtain the high temperature limit of the change in the free energy, $\Delta A^{T}$, of a scalar field in a box of dimension $l_{1} \times l_{2} \times L$, as we move an additional wall from a position at a distance $l_{3}$ from one of the $l_{1}$ by $l_{2}$ sides to the middle of the box at $L / 2$. Thus, from Eq. (20), we have

$$
\Delta A_{\text {class }}^{T}=k_{B} T \mathcal{A} \int \frac{d^{2} k_{\perp}}{(2 \pi)^{2}} \frac{1}{2} \sum_{n=-\infty}^{\infty}\left[\ln \frac{\omega\left(\pi n / l_{3}, k_{\perp}\right)}{\omega\left(2 \pi n / L, k_{\perp}\right)}+\ln \frac{\omega\left(\pi n /\left[L-l_{3}\right], k_{\perp}\right)}{\omega\left(2 \pi n / L, k_{\perp}\right)}\right]
$$

where $\omega\left(k_{3}, k_{\perp}\right)=c \sqrt{k_{3}^{2}+k_{\perp}^{2}}$, with $k_{3}=\pi n / \bar{l}_{3}$ where $\bar{l}_{3}$ is the appropriate distance from the wall. Using the integral representation

$$
\ln [a / b]=\frac{1}{2} \int_{0}^{\infty} \frac{d \lambda}{\lambda}\left[e^{-\lambda b^{2}}-e^{-\lambda a^{2}}\right],
$$

for the logarithms in Eq. (21), the integration over $k_{\perp}$ can readily be performed on using polar coordinates. In addition we use the reflection property,

$$
\sum_{n=-\infty}^{\infty} e^{-n^{2} \pi^{2} x}=(\pi x)^{-1 / 2} \sum_{n=-\infty}^{\infty} e^{-n^{2} / x}
$$

to rewrite the resulting sums over $n$. [ Eq. (23) and Eq. (12) both relate the values of a function at the argument $x$ and at the "reflected" argument $1 / x$. Eq. (23) is a particular case of Poisson's summation formula.] We then obtain

$$
\frac{\Delta A_{\text {class }}^{T}}{k_{B} T \mathcal{A}}=-\frac{1}{16 \pi \sqrt{\pi}} \sum_{n=-\infty}^{\infty} \int_{0}^{\infty} \frac{d \lambda}{\lambda^{5 / 2}}\left[l_{3} e^{-\frac{n^{2} l_{3}^{2}}{\lambda}}+\left(L-l_{3}\right) e^{-\frac{n^{2}\left(L-l_{3}\right)^{2}}{\lambda}}-L e^{-\frac{n^{2}(L / 2)^{2}}{\lambda}}\right] .
$$

The $n=0$ contribution vanishes and $\sum_{n=-\infty}^{\infty}$ reduces to $2 \sum_{n=1}^{\infty}$. The integrals with $n \neq 0$ converge term by term; with

$$
\int_{0}^{\infty} \frac{d \lambda}{\lambda^{5 / 2}} e^{-\alpha / \lambda}=\frac{\pi^{1 / 2}}{2 \alpha^{3 / 2}}
$$

we thereby arrive at

$$
\Delta A_{\text {class }}^{T}=-k_{B} T \mathcal{A} \frac{1}{16 \pi} \zeta(3)\left[\frac{1}{l_{3}^{2}}+\frac{1}{\left(L-l_{3}\right)^{2}}-\frac{8}{L^{2}}\right],
$$

which for $L \gg l_{3}$ is the leading term of Eq. (15) up to a factor of 2; the factor appears because we considered the scalar case here.[We strongly suspect that there may be a simpler classical derivation of Eq. (26) than the one given here.]

A related problem is that of the Casimir energy, at $T=0$, of two spinless charged particles in free space. There is a classical contribution in that case too, but it is not the leading term and the question arises if the term can be evaluated using classical theory. The answer in this case is also positive 25]. 
An interesting point is that on dimensional grounds the absence of $\hbar$ leads to the absence of $c$, though we are studying electromagnetic waves. There is no contradiction; the classical Maxwell equations have meaning in the nonrelativistic $(c \rightarrow \infty)$ limit [26]. Thus, for example, in the dipole approximation the classical Maxwell equations give a $c$-independent radiative transition rate.

\section{THE SCALAR FIELD}

In App. B we prove the validity of Eq. (8) by making a connection with the results of 13]. We here give an ab initio proof of that equation for a massless scalar field. The relation to the electromagnetic case is discussed in App. C (in a less broad context than that given, for example, in ref.[16]).

Thus, to substantiate some of the arguments of the main text, we consider a free massless scalar field $\phi$ that satisfies Dirichlet boundary conditions

$$
\phi\left(x_{i}=0\right)=\phi\left(x_{i}=l_{i}\right)=0,
$$

at the boundaries of a box with spatial dimensions $l_{1} \times l_{2} \times \ldots \times l_{D}$. The box serves as an infrared cutoff and guaranties a discrete spectrum. The dimension $D$ of the box is arbitrary and can be used to dimensionally regularize the ultraviolet behavior of subsequent expressions by analytic continuation to non-integer dimensions. Additional boundary conditions may be imposed on the field inside the box. One could, for instance, demand that the field also vanish on a spherical shell located within the box, or, more appropriately for the Casimir force between plates, that it also vanish on a plane within the box.

The free energy $A^{T}$ is related to the partition function $Z$ by $A^{T}=-\beta^{-1} \ln Z$, where $\beta=1 /\left(k_{B} T\right)$. Formally, $Z$ is

$$
Z=\operatorname{Tr} e^{-\beta H}
$$

where $H=\sum_{\mathbf{k}} E_{\mathbf{k}}\left(a_{\mathbf{k}}^{\dagger} a_{\mathbf{k}}+1 / 2\right)$ is the free hamiltonian. $a_{\mathbf{k}}\left(a_{\mathbf{k}}^{\dagger}\right)$ destroys (creates) a quantum with quantum numbers $\mathbf{k}$ in the (possibly rather complicated) cavity. Crucial for the following is that $H$ [ignoring any (weak) self-coupling of the scalar field] commutes with the number operators $n_{\mathbf{k}}=a_{\mathbf{k}}^{\dagger} a_{\mathbf{k}}$ no matter how complicated the boundary conditions on the field may be. $H$ can therefore be diagonalized in a basis that enumerates the number of quanta in each cavity mode and has the eigenvalues $\sum_{\mathbf{k}} E_{\mathbf{k}}\left(n_{\mathbf{k}}+1 / 2\right)$ with each $n_{\mathbf{k}}$ a positive integer or 0. Evaluating the trace of Eq. (28) in this number basis, one has

$$
\ln Z=\ln \left[\prod_{\mathbf{k}} \sum_{n_{\mathbf{k}}=0}^{\infty} e^{-\beta\left(n_{\mathbf{k}}+1 / 2\right) E_{\mathbf{k}}}\right]=-\sum_{\mathbf{k}} \ln \left[2 \sinh \left(\beta E_{\mathbf{k}} / 2\right)\right] .
$$

Instead of computing the free energy or partition function directly, it will be convenient to first consider the energy at temperature $T$,

$$
\mathcal{E}^{T}=\frac{\partial}{\partial \beta}\left(\beta A^{T}\right)=-\frac{\partial}{\partial \beta} \ln Z .
$$

Differentiating Eq. (29), one finds

$$
\mathcal{E}^{T}=\sum_{\mathbf{k}} E_{\mathbf{k}}\left[\frac{1}{2}+\sum_{m=1}^{\infty} e^{-m \beta E_{\mathbf{k}}}\right] .
$$


For $E_{\mathbf{k}}>0$ one can use Cauchy's theorem with a contour that runs just above the real axis and is closed by a large semicircle in the upper complex plane and write

$$
E_{\mathbf{k}}\left[\frac{1}{2}+\sum_{m=1}^{\infty} e^{-m \beta E_{\mathbf{k}}}\right]=-\lim _{\epsilon \rightarrow 0^{+}} \frac{1}{\pi} \int_{-\infty+i \epsilon}^{\infty+i \epsilon} \frac{\lambda^{2} d \lambda}{E_{\mathbf{k}}^{2}+\lambda^{2}}\left[1 / 2+\sum_{m=1}^{\infty} e^{i m \beta \lambda}\right],
$$

up to terms that do not depend on $E_{\mathbf{k}}$ nor on $\beta$; in Eq. (32) we have dropped the contribution from the semicircular part of the contour. (For a sufficiently large radius $\Omega$ of the contour, this contribution is $\Omega / \pi$ up to terms that vanish for $\Omega \rightarrow \infty$.) The integral along the semicircular part of the contour thus does not depend on $E_{\mathbf{k}}$ nor $T$ and can be absorbed in the overall normalization of the energy. Such constant contributions are irrelevant for the discussion of energy differences. With $\lambda=x+i \epsilon$ and $x$ real, the term on the right hand side of Eq. (32), in square brackets, is 27]

$$
Q \equiv\left[1 / 2+\sum_{m=1}^{\infty} e^{i m \beta(x+i \epsilon)}\right]=\frac{i}{2} \cot (\beta x / 2)+\pi \sum_{n=-\infty}^{\infty} \delta(\beta x-2 \pi n)+O(\epsilon) .
$$

We can now evaluate the integral in Eq. (32). Apart from terms of order $\epsilon$ that vanish in the limit $\epsilon \rightarrow 0^{+}$, we can replace $\lambda$ by $x$. Because $\cot (\beta x / 2)$ is an odd function of $x$, the imaginary part of $Q$ does not contribute to the integral. (This reflects the assumption that the cavity is ideal.) Formally, the energy of an ideal cavity at finite temperature is thus, from Eqs. (31), (32), and (33),

$$
\begin{aligned}
\mathcal{E}^{T} & =-\sum_{\mathbf{k}} \sum_{n=-\infty}^{\infty} \frac{(2 \pi n)^{2}}{\beta^{3}} E^{-2}(\mathbf{k}, n)=-\frac{\partial}{\partial \beta} \sum_{\mathbf{k}} \sum_{n=-\infty}^{\infty} \ln E(\mathbf{k}, n) \\
& =-\frac{\partial}{\partial \beta} \ln \left[\prod_{\mathbf{k}} \prod_{n=-\infty}^{\infty} E^{-1}(\mathbf{k}, n)\right]
\end{aligned}
$$

where

$$
E(\mathbf{k}, n)=\left[E_{\mathbf{k}}^{2}+(2 \pi n / \beta)^{2}\right]^{1 / 2} .
$$

Since $\mathcal{E}^{T}=-(\partial / \partial \beta) \ln Z$, Eq. (34) determines the partition function up to an overall normalization, or equivalently, the entropy up to an overall constant (which can be determined by demanding that the entropy vanish at $T=0$ ). Eq. (34) formally shows that the partition function is proportional to the square root of the inverse determinant of the differential operator $H_{4}^{2}=h^{2}-(\hbar c)^{2} \frac{\partial^{2}}{\partial x_{T}^{2}}$, where $x_{T}$ is the additional Euclidean coordinate in which the fields are periodic (with periodic length $l_{T}$ ) and $h$ is the differential operator (single particle hamiltonian) with eigenvalues $E_{\mathbf{k}}$. The positive eigenvalues of $H_{4}$, the $E(\mathbf{k}, n)$ given in Eq. (35), are the allowed energies of a massless scalar in the four-dimensional cavity constructed by augmenting every point of the original three-dimensional cavity by a circle of circumference $l_{T}$, that is, the 4 -dimensional space is the tensor product of the cavity and a circle whose circumference is proportional to the inverse temperature. From Eq. (34) and Eq. (30) we see that the free energy of the cavity can be written formally as

$$
A_{\text {cavity }}^{T}=\beta^{-1} \sum_{\mathbf{k}} \sum_{n=-\infty}^{\infty} \ln E(\mathbf{k}, n)=\frac{\hbar c}{l_{T}} \int_{0}^{\infty} d E^{\prime} \rho_{\text {cavity }}^{T}\left(E^{\prime}\right) \ln \left(E^{\prime}\right),
$$


with

$$
\rho_{\text {cavity }}^{T}\left(E^{\prime}\right)=\sum_{\mathbf{k}} \sum_{n=-\infty}^{\infty} \delta\left(E(\mathbf{k}, n)-E^{\prime}\right) .
$$

Eq. (37) shows that $\rho_{\text {cavity }}^{T}\left(E^{\prime}\right)$ is the spectral density of the four-dimensional cavity constructed from the three-dimensional one in the above manner. Changes of $\rho_{\text {cavity }}^{T}\left(E^{\prime}\right)$ due to (adiabatic) deformation of the cavity, including a change of temperature, can then be approximated semi-classically using periodic classical rays of the four-dimensional cavity. This leads to a description of the free energy in terms of periodic paths in the four-dimensional cavity which we exploited when we guessed at the form of the free energy in Sec. 2.

To be somewhat more concrete, consider as an example a cubic cavity with sides of length $\bar{l}$. The momentum components for the mode with energy $E_{\mathbf{k}}$ are $k_{i} \hbar=n_{i} \pi \hbar / \bar{l}$, and the energies in the corresponding four-dimensional cavity at finite temperature are

$$
E(\mathbf{k}, n)=\pi \hbar c\left[\sum_{i=1}^{3}\left(\frac{n_{i}}{\bar{l}}\right)^{2}+\left(\frac{n}{l_{T} / 2}\right)^{2}\right] ;
$$

as always, the length associated with the fourth (periodic) dimension, the analog of the lengths associated with the spatial dimensions, is $l_{T} / 2$.

Note that Eq. (36) is formal in two respects: the integral over the energy diverges due to the ultraviolet behavior of the spectral density, and Eq. (36) specifies the free energy only up to (a generally similarly divergent) contribution proportional to $T$. Considerably more rigorous and physically relevant expressions can be obtained from Eq. (36) for changes in the free energy due to adiabatic deformations of the three-dimensional cavity. In physical terms the difference in the free energy is work that has to be done to change the boundary conditions for the field and should therefore be finite whenever it is physically feasible to do so. The ambiguity that Eq. (36) defines the entropy only up to a constant that does not depend on the temperature is removed by requiring that the entropy vanish at $T=0$.

Let us finally remark that for $T \rightarrow 0$ and $E_{\mathbf{k}}$ fixed,

$$
\lim _{\beta \rightarrow \infty} \sum_{n=-\infty}^{\infty} \beta^{-1} \ln \left[E_{\mathbf{k}}^{2}+(2 \pi n / \beta)^{2}\right]^{1 / 2}=E_{\mathbf{k}} / 2
$$

up to an infinite constant that does not depend on $E_{\mathbf{k}}$ and therefore cancels in differences. [ Eq. (39) follows upon subtracting the $E_{\mathbf{k}}$-independent term $\ln \left[\mu^{2}+(2 \pi n / \beta)^{2}\right]^{1 / 2}$ from the summand in Eq. (39) and noting that the so regularized sum defines a convergent integral in the limit $\beta \rightarrow 0$ with the value $\left(E_{\mathbf{k}}-\mu\right) / 2$. The dependence on the subtraction point $\mu$ can be absorbed in the overall energy normalization and cancels in energy differences.] At $T=0$ Eq. (36) thus gives the usual expression for the Casimir energy. The classical periodic orbits of the four-dimensional cavity in this limit are just the periodic orbits of the three-dimensional cavity, since any path that winds about the fourth dimension becomes arbitrarily long as $T \rightarrow 0$. Note that whereas the spectral density $\rho_{\text {cavity }}^{T}$ generally is proportional to the four-dimensional volume $l_{T} V_{\text {cavity }}$ the factor $1 / l_{T}$ in Eq. (36) ensures that $A^{T}$ generally will only be 
proportional to the three-dimensional volume of the cavity (which we extracted explicitly in Sec. 2).

Although our starting point, Eq. (34) for the free energy, is the field theoretic one[14], the evaluation of this expression using the spectral density of a massless scalar in four spatial dimensions and, further, the semiclassical approximation to (changes in) this spectral density using classical periodic rays of the four-dimensional cavity is perhaps a bit unusual. This sketch of the relation between the free energy and the periodic classical rays of a corresponding fourdimensional cavity is the mathematical and perhaps even the physical basis for our guess of the free energy of two parallel plates in Sec. 2.

\section{DISCUSSION}

For ideal parallel plates, the semiclassical periodic orbital formalism gives a result for $\mathcal{E}_{\text {Cas }}$ different in form but equivalent to that obtained by other methods. However, the approach provides a simpler physical picture, allows a simpler transition from $\mathcal{E}_{\mathrm{Cas}}$ to $A^{T}$, and gives a form simpler to evaluate numerically. It evaluates changes in the free energy due to changes in the temperature and in the cavity boundaries using the periodic classical paths in a four-dimensional cavity which is the tensor product of the original three-dimensional one and a circle. In a number of instances this amounts to replacing the contribution to the Casimir energy of each periodic classical path of length $L_{\gamma}$ at $T=0$ by the sum of contributions of periodic classical paths of length $L_{\gamma}^{T}\left(n_{T}\right)=\left[L_{\gamma}^{2}+\left(n_{T} l_{T}\right)^{2}\right]^{1 / 2}$ that, in addition, wind about the extra fourth dimension $n_{T}$ times.

Furthermore, preliminary research indicates that this considerable simplification may be present for a number of geometries, for scalar as well as for electromagnetic fields, and, at least for parallel plates, for dielectric walls. The simplification is a slight extension of the domain of applicability of Gutzwiller's semi-classical periodic orbit approach, allowing the method to be used for a number of systems at finite temperature. Generalizing the result for parallel plates, it appears that the periodic classical paths of various lengths can be interpreted as dual variables to the eigenfrequencies of a cavity: in rectangular cavities the two descriptions are related by Poisson's summation theorem.

\section{ACKNOWLEDGEMENTS}

This work was supported by the National Science Foundation Grant PHY0070525. M.S. greatly enjoyed the hospitality of New York University where most of this work was done.

\section{APPENDIX A: FINITE TEMPERATURE AND THE INTRODUCTION OF A FOURTH DIMENSION}

As a vague indication of why the temperature can be treated by introducing a periodic fourth dimension, compare the standard operators $\exp (-i H t / \hbar)$ in quantum theory and $\exp (-\beta H)$ in statistical mechanics. One finds $\beta=i t / \hbar$. The presence of $i$ suggests periodicity, and we therefore drop the factor $i$ and 
replace $t$ by a period $\tau$, giving $\beta=\tau / \hbar$. For photons, we can replace $\tau$ by $l_{T} / c$, leading to $l_{T}=\hbar c / k_{B} T$. For parallel plates, $F / A$ must be proportional to $l_{1} l_{2}$, and the problem reduces to the determination of a function of $l_{3}$ and $l_{T}$. If one introduces periodic orbits between the plates, then the appropriate surface in $l_{3}$ and $l_{T}$ is the surface of a cylinder of length $l_{3}$ and circumference $l_{T}$. Periodic classical paths involve lengths $2 n_{3} l_{3}$, for $n_{3}$ to and fro motions between the plates, and $n_{T} l_{T}$, for circling $n_{T}$ times around the cylinder. Since the coordinate system is Cartesian, the length of the shortest orbit that circles the cylinder $n_{T}$ times and goes back and forth between the plates $n_{3}$ times is just Eq. (4). For a proper derivation of the introduction of a fourth dimension, see 14] and Sec. 5.

\section{APPENDIX B: AGREEMENT WITH A PRE- VIOUS RESULT}

We now show that $F^{T} / \mathcal{A}$ as given by Eq. (9) agrees with the force per unit area obtained previously 13]. Using Eqs.(3.8), (3.10), (3.11), (3.12), line 2 of (3.13), and (3.14) of 13., we find

$$
\frac{F^{T}}{\mathcal{A}}=-\frac{1}{4 \beta l_{3}^{3}} \sum_{n=-\infty}^{\infty} c\left(n \hbar c /\left(2 k_{B} T l_{3}\right)\right),
$$

where

$$
c(\alpha)=-\frac{\beta}{4 \pi^{2} \alpha l_{3}} \frac{d^{2}}{d \alpha^{2}} J(\alpha)
$$

with

$$
J(\alpha)=\int_{0}^{\infty} d y \frac{\sin (\alpha y)}{e^{y}-1} .
$$

We obtain a different form for $c(\alpha)$ from that obtained in 13 by expanding the denominator of Eq. (B3),

$$
J(\alpha)=\operatorname{Im} \sum_{m=1}^{\infty} \int_{0}^{\infty} d y e^{(i \alpha-m) y}=\sum_{m=1}^{\infty} \frac{\alpha}{m^{2}+\alpha^{2}},
$$

in agreement with Eq. (13). Performing the differentiation leads to

$$
c(\alpha)=\frac{\beta}{2 \pi^{2} l_{3}} \sum_{m=1}^{\infty} \frac{3 m^{2}-\alpha^{2}}{\left[m^{2}+\alpha^{2}\right]^{3}} .
$$

Insertion of Eq. (B5) with $\alpha=n \hbar c /\left(2 k_{B} T l_{3}\right)=n l_{T} /\left(2 l_{3}\right)$ in Eq. (B1) reproduces Eq. (9).

\section{APPENDIX C: ELECTROMAGNETIC VER- SUS SCALAR FIELDS}

We are primarily interested in electromagnetic fields and we therefore comment on the relationship between the free energy $A_{s c}^{T}$ of a scalar field and the free 
energy of an electromagnetic field, denoted, as elsewhere, by $A^{T}$. The boundary conditions for scalar and electromagnetic fields are different, and further, for the latter case, both the condition $\operatorname{div} \mathbf{E}=0$, which has no scalar field analog, and polarization must be accounted for; these three differences are not, of course, unrelated. Electrodynamics assumes different forms in spaces of a different number $D$ of spatial dimensions and we will consider only $D=3$. We will further limit our considerations to a rectangular box, with arbitrary dimensions $l_{1}$ by $l_{2}$ by $l_{3}$.

As is well known, the condition $\operatorname{div} \mathbf{E}=0$ reduces the number of independent components of $\mathbf{E}$ to at most two and for a perfectly conducting box gives conditions on the quantum numbers $n_{1}, n_{2}$ and $n_{3}$ of the modes. There are two independent components if none of the $n_{i}$ vanish, one if one of the $n_{i}$ vanishes, and none if two of the $n_{i}$ vanish. In the periodic orbital approach one considers classical ray's; in this case the condition $\operatorname{div} \mathbf{E}=0$ implies certain phase changes of each of the two independent polarizations upon reflection.

We consider polarization effects for three cases, i) $l_{1} \gg l_{3}$ and $l_{2} \gg l_{3}$, ii) $l_{1} \gg l_{2}$ and $l_{1} \gg l_{3}$, and iii) arbitrary values of $l_{1}, l_{2}$ and $l_{3}$. For case i), the orbitals pass back and forth between, and perpendicular to, the two $l_{1}$ by $l_{2}$ walls, the two states of polarization behave identically, and the effective electromagnetic response function, $\delta g_{e l}$, is twice that of the relevant scalar response function $\delta g_{s c}$. For case ii), the situation is the same for orbitals which pass back and forth between the $l_{1}$ by $l_{3}$ or the $l_{2}$ by $l_{3}$ walls. For orbitals which reflect off four walls, the pair of $l_{1}$ by $l_{3}$ and the pair of $l_{2}$ by $l_{3}$ walls, the polarization vector $\hat{\epsilon}$ is unchanged on reflection for $\hat{\epsilon}$ perpendicular to the plane of scattering; $\hat{\epsilon}$ is changed on reflection for $\hat{\epsilon}$ in the plane of scattering, but returns to its original value at the end of the (planar) periodic orbit, and once again we have, effectively, $\delta g_{e l}=2 \delta g_{s c}$. The situation is quite different for case iii), since periodic orbits that reflect off all six walls are not planar. [Periodic rays that reflect off all six walls cannot be planar, since no plane can cut all surfaces of the rectangular three dimensional volume.] To visualize one such non-planar orbit consider a classical ray that is close to and parallel to one of the spatial diagonals of the parallelepiped - not contained in any of the surfaces - and lies in one of the diagonal planes that contains the diagonal and two of the corners of the parallelepiped not connected by the diagonal. After reflection off all three walls at one of the corners, the ray, for symmetry reasons, is again parallel to the diagonal of the parallelepiped and in the same plane as before reflection at the corner. After reflecting off the other three walls at the diagonally opposite corner, the path of the ray closes to a periodic one. If the initial ray is chosen very close to the diagonal, the length of this periodic orbit at the very least is close to twice the length of the diagonal of the parallelepiped, i.e., $L(1,1,1)=\left[\left(2 l_{1}\right)^{2}+\left(2 l_{2}\right)^{2}+\left(2 l_{3}\right)^{2}\right]^{1 / 2}$. The length of the periodic classical ray that reflects once off each pair of parallel walls in reality does not depend on how close to the diagonal the incident ray is chosen and thus is twice the length of the diagonal. The calculation would be much more complicated if that were not the case. More complicated non-planar periodic paths, that reflect a different number of times off each pair of parallel surfaces are obtained by changing the initial direction of the ray. For non-planar orbits there is a mixing of the two states of polarization and $\delta g_{e l}$ generally is not just $2 \delta g_{s c}$; the contribution to $\delta g_{e l}$ of non-planar orbits is proportional to the trace of an orthogonal two-by-two polarization matrix. The existence of non-planar periodic orbits for case iii), 
and the consequent non-diagonality of the polarization matrix, make less surprising the fact that $\mathcal{E}_{\text {Cas }}$ in case iii) depends 28 not only on the 3-dimensional path lengths,

$$
L\left(n_{1}, n_{2}, n_{3}\right)=\left[\left(2 l_{1} n_{1}\right)^{2}+\left(2 l_{2} n_{2}\right)^{2}+\left(2 l_{3} n_{3}\right)^{2}\right]^{1 / 2},
$$

but also on the one-dimensional path lengths $L\left(n_{i}\right)=2 l_{i} n_{i}$ for $i=1, \ldots, 3$. The presence of one-dimensional paths can also be associated with the restriction of the quantum numbers due to the boundary conditions. Thus for scalar fields satisfying Dirichlet boundary conditions in a rectangular cavity - the eigenfunctions are products of sines - none of the $n_{i}$ quantum numbers vanish. Finally, we note that we stated in 15 that the semiclassical approach was exact if any of the ratios formed from $l_{1}, l_{2}$ and $l_{3}$ was arbitrarily large, as is the case for i) and ii) but not for iii). The restriction was necessary because we had assumed that $\delta g_{e l}=2 \delta g_{s c}$.

We remark, parenthetically, that the four dimensional space characterized by $l_{1}, l_{2}, l_{3}$ and $l_{T}$, with three spatial dimensions and one periodic dimension (with a circumference proportional to the inverse temperature), is not equivalent to electrodynamics in four spatial dimensions; $\operatorname{div} \mathbf{E} \neq 0$ for the latter space.

\section{References}

[1] H.B.G. Casimir, Proc. K. Ned. Akad. Wet.51, 793 (1948).

[2] P.W. Milonni, The Quantum Vacuum, (Academic, New York, 1993). See especially Chaps. 7 and 8.

[3] V.M. Mostepanenko and N.N. Trunov, The Casimir Effect and its Applications, (Clarendon Press, Oxford, 1997).

[4] S.K. Lamoreaux, Resource Letter, Am.J.Phys. 67, 850 (1999).

[5] Comments on Modern Physics: Comments on Atomic and Molecular Physics D1 171 (2000). This special issue, with guest editors J.F. Babb, P.W. Milonni and L. Spruch, is exclusively devoted to Casimir effects.

[6] See the contribution by S. Lamoreaux in [5] and references therein to his previous work.

[7] See the contribution by U. Mohideen and A. Roy in [5]; see also B.W. Harris, F. Chen and U. Mohideen, Phys. Rev. A62, 052109 (2000).

[8] H.B. Chan, V.A. Aksyuk, D.J. Bishop and F. Capasso, Quantum Mechanical Actuation of Micromechanical Systems by the Casimir Force, BellLaboratory Preprint (12/00).

[9] B.V. Derjaguin and I.I. Abrikosova, Sov. Phys. JETP3, 819 (1957); B.V. Derjaguin, I.I. Abriksova and E.M. Lifshitz, Quarterly Review 10, 295 (1968); M.J. Sparnaay, Physica 24, 751 (1958); J.N. Israelachvili and D. Tabor,Proc. R. Soc. London A331, 19 (1972); see also ref.[2], p.272.

[10] M. Schaden and L. Spruch, Phys. Rev. Lett. 84, 459 (2000). 
[11] L.H. Ford and N.F. Svaiter, Phys. Rev. A62, 062105 (2000).

[12] E.M. Lifshitz, Sov. Phys. JETP2, 73 (1956).

[13] J. Schwinger, L.L. de Raad, Jr. and K.A. Milton, Ann. Phys.115, 1 (1978).

[14] J.I. Kapusta, Finite-Temperature Field Theory (Cambridge Univ. Press, Cambridge 1989), Chapters 1 and 2.

[15] M. Schaden and L. Spruch, Phys. Rev. A58, 935 (1998). See also|55.

[16] A. Balian and B. Duplantier, Ann. Phys. (N.Y.) 104, 300 (1977);ibid112, 165 (1978).

[17] M.C. Gutzwiller,Chaos in Classical and Quantum Mechanics (Springer, New York,1990); see also L.E.Reichl, The Transition to Chaos (Springer, New York, 1992), Chap.8.

[18] This theorem was first used to compute Casimir energies by N.G. van Kampen, B.R.A. Nijboer and K. Schramm, Phys. Lett. A26, 307 (1968). See also[2].

[19] One of the many applications of the theorem is that of F. Zhou and L. Spruch, Phys. Rev. A52, 297 (1995), to the study of sets of parallel walls of arbitrary thickness and spatially independent but otherwise arbitrary permittivity.

[20] L.S. Brown and G.I. Maclay, Phys. Rev. 184, 1272 (1969), were the first to recognize that there was a symmetry, or duality, between high and low temperatures.

[21] P.M. Morse and H. Feshbach, Methods of Theoretical Physoics (McGraw Hill, New York, 1953) p.467.

[22] F. Sauer, dissertation, Göttingen, 1962 (unpublished); J. Mehra, Physica 37, 145 (1967); M.L.Levin and S. M. Rytov, The Theory of Thermal Equilibrium Fluctuations in Electrodynamics (Nauka, Moscow, 1967).

[23] V.B. Svetovoy and M.V. Lokhanin, Mod. Phys. Lett. A15, 1013 (2000);ibid,1437 (2000).

[24] E.A. Power, Introductory Quantum Electrodynamics (Elsevier,New York,1964). Appendix I.

[25] J. Sucher,Comments At. Mol. Phys. D1, 227 (2000);Phys. Rev. D49, 4284 (1994); Comments At. Mol. Phys. 30, 129 (1994); J. Sucher and G. Feinberg, in Long Range Casimir Forces: Theory and Recent Experiments in Atomic Systems, edited by F.S. Levin and D.A. Micha (Plenum, New York, 1993).

[26] M. Lebellac and J.M. Levy-LeBlond, Nuovo Cimento B14, 217 (1973). See also R. Shakeshaft and L. Spruch, Phys. Rev. Lett. 38, 175 (1977). 
[27] Ignoring corrections of order $\epsilon, \operatorname{Im} Q$ follows by evaluating the geometric series; $\operatorname{Re} Q$ follows by writing it as $\pi \sum_{-\infty}^{\infty} \phi_{m}(y) \phi_{m}^{*}(-y)$, where $y=\beta x / 2$ and $\phi_{m}(y)=(2 \pi)^{-1 / 2} \exp ($ imy $)$, and using completeness of these functions on the interval $(2 n-1) \pi \leq y<(2 n+1) \pi$ for any $n$.

[28] W. Lukosz, Physica (Amsterdam) 56, 109 (1971). 